\title{
Haptics with Input: Back-EMF in Linear Resonant Actuators to Enable Touch, Pressure and Environmental Awareness
}

\author{
Artem Dementyev \\ Google Research \\ Mountain View, CA \\ artemd@google.com
}

\author{
Alex Olwal \\ Google Research \\ Mountain View, CA \\ olwal@acm.org
}

\author{
Richard F. Lyon \\ Google Research \\ Mountain View, CA \\ dicklyon@google.com
}

\begin{abstract}
Today's wearable and mobile devices typically use separate hardware components for sensing and actuation. In this work, we introduce new opportunities for the Linear Resonant Actuator (LRA), which is ubiquitous in such devices due to its capability for providing rich haptic feedback. By leveraging strategies to enable active and passive sensing capabilities with LRAs, we demonstrate their benefits and potential as self-contained I/O devices. Specifically, we use the back-EMF voltage to classify if the LRA is tapped, touched, as well as how much pressure is being applied. The back-EMF sensing is already integrated into many motor and LRA drivers. We developed a passive low-power tap sensing method that uses just $37.7 \mu \mathrm{A}$. Furthermore, we developed active touch and pressure sensing, which is low-power, quiet ( $2 \mathrm{~dB})$, and minimizes vibration. The sensing method works with many types of LRAs. We show applications, such as pressure-sensing side-buttons on a mobile phone. We have also implemented our technique directly on an existing mobile phone's LRA to detect if the phone is handheld or placed on a soft or hard surface. Finally, we show that this method can be used for haptic devices to determine if the LRA makes good contact with the skin. Our approach can add rich sensing capabilities to the ubiquitous LRA actuators without requiring additional sensors or hardware.
\end{abstract}

\section{Author Keywords}

Linear resonant actuator; sensing; haptic actuator; back-EMF; touch; pressure

\section{CCS Concepts}

-Human-centered computing $\rightarrow$ Haptic devices;

\section{INTRODUCTION}

As our wearable and handheld devices are becoming smaller, haptic feedback provides a vital channel of communication [27]. Researchers have been exploring and documenting the benefits of using haptic feedback for quite some time, and

\footnotetext{
Permission to make digital or hard copies of part or all of this work for personal or classroom use is granted without fee provided that copies are not made or distributed for profit or commercial advantage and that copies bear this notice and the full citation on the first page. Copyrights for third-party components of this work must be honored. For all other uses, contact the owner/author(s).

UIST'20, October 20-23, 2020, Virtual Event, USA

(C) 2020 Copyright held by the owner/author(s).

ACM ISBN 978-1-4503-7514-6/20/10.
}

DOI: https : //doi .org/10.1145/3379337. 3415823

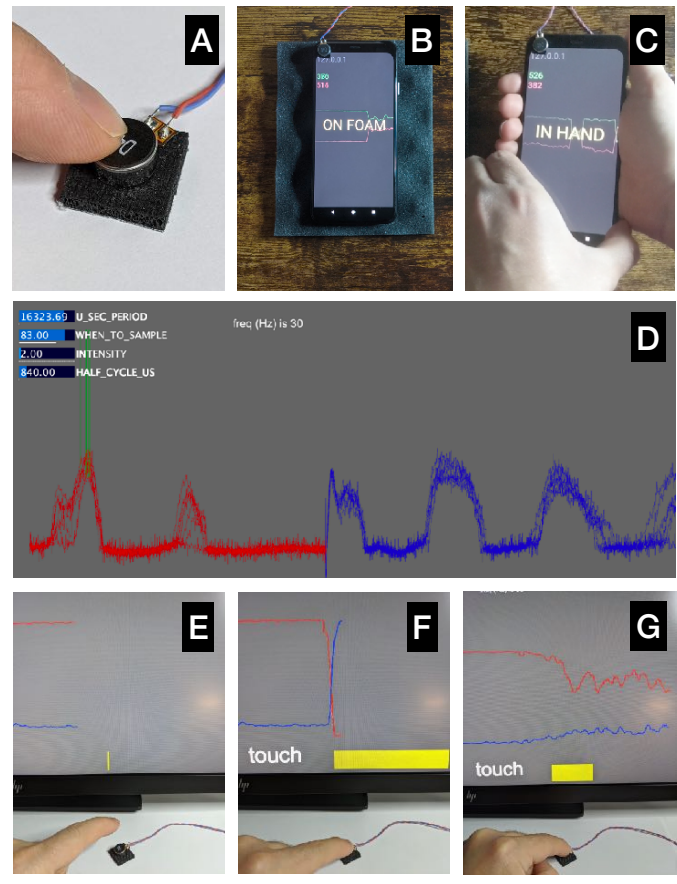

Figure 1. Using the LRA as a sensor. A) Close-up of the LRA, mounted on a piece of foam. B) and C) The LRA attached to the phone to detect if the phone is on foam or handheld. D) The user interface for tuning and displaying raw back-EMF signals. The red and blue lines display the real-time back-EMF signals from high (red) and low (blue) half cycles. E) Time series, extracted from raw back-EMF, before touching the LRA. F) Touch is detected. G) The LRA is also pressure-sensitive, with magnitude indicated by the yellow bar.

they are now ubiquitous in nearly all wearable devices and mobile phones.

The linear resonant actuator (LRA) is one of the critical enablers of haptic feedback. The LRA is a linear electromagnetic motor that leverages resonance to provide strong haptic feedback in a small package.

While integrated sensing and actuation has been explored in research projects $[22,35,23]$, touch, and pressure sensing tend to depend on additional hardware, separate from the haptic actuator. For example, a mechanical button will require an independent sensor and actuator. 
In this work, we demonstrate that the widely available LRA actuator can be driven to also sense a wide range of external information, such as touch, tap and pressure, in addition to being able to relay information about contact with the skin, objects and surfaces. We achieve this with unmodified LRAs by multiplexing the actuation with short pulses of custom waveforms that are designed to enable sensing using the backEMF voltage. This approach was, in part, inspired by previous works where commodity components, such as LEDs, were extended to also support input $[13,8]$.

We demonstrate the potential of this approach with configurations where the LRA is mechanically decoupled from the chassis, which, for example, enables expressive discrete buttons and vibrotactile interfaces. We also show how the approach can be applied to configurations with chassis-coupled LRAs, which could bring rich sensing opportunities to integrated haptics modules in mobile devices. Our technique is potentially compatible with many LRA drivers $[32,15]$ as they already employ back-EMF sensing for autotuning of the vibration frequency.

The use of the LRA as a sensor poses unique challenges that we had to solve, since back-EMF requires active sensing based on actuation. Our problem was to allow continuous sensing while minimizing vibrations, sound, and power consumption. Our insight was that we identified the opportunity to design a custom, off-resonance driver waveform. We also show that the LRA can be used as a passive wake-up tap sensor, where the back-EMF voltage is generated by contact and pressure.

The contributions of this work are as following:

1. A technique for extending LRAs for active and passive sensing of contact. Our approach is applicable to unmodified LRAs, as the back-EMF sensing method is already employed in many commercial drivers.

2. Technical characterization of power consumption, vibration, and touch sensitivity.

3. Applications that demonstrate mechanically uncoupled and coupled interactive devices, such as tap-, touch-, and pressure-sensitive buttons, and mobile and wearable devices that can detect their surroundings.

\section{RELATED WORK}

In this section, we will describe previous work in commercial haptic technologies, as well as in research.

\section{Mobile haptic technologies}

Early haptic technology, developed in the 1970s, leveraged an eccentric rotating mass (ERM). Such technology became widely used in early mobile phones and pagers, as well as other devices. The ERM typically uses a brushed DC motor with an unbalanced mass attached to the motor. This eccentric mass creates strong vibrations, but cannot be used for fine-grained haptics due to its slow response. The advantage of the ERM actuator is its simplicity and low cost. The disadvantages are numerous, including loud noise, large inertia, and the wear of the moving parts.
Motivated by the limitations of the ERM, more sophisticated haptic actuators were developed. Most significantly, they have largely been replaced in mobile devices by LRAs, such as the variant named Taptic Engine used in Apple's iPhone and iWatch [9].

Voice coils were originally designed to produce audible sound, but have also been applied to actuation. One such example is the high-end Haptuator device [18], which has been used in several research projects, such as Grabity [6]. Voice coils are similar to LRAs but are not designed to operate at resonance. Their emphasis on audible sound required their support for a broad frequency range, making them bulkier and less efficient than LRAs for producing force.

Piezoelectric actuators have shown promise to be widely adopted for haptics, given the possibility for miniaturization and fast response. Haptic piezo actuators are usually designed as two-layer bimorphs, which bend when a large voltage is applied (typically 50-200 V). There are commercially-available piezoelectric haptic actuators, such as the TDK PowerHap series [36]. The disadvantage of piezoelectric actuators is their high cost, small displacement, high voltage requirements, and limited use in mass-market products for haptics.

\section{Touch screens and haptic feedback}

There have been many successful attempts to integrate touch sensing and tactile feedback on mobile touchscreens or touch panels. TeslaTouch [3], for example, used electrovibrations to create tactile feedback on a capacitive touch screen, but requires a return ground path for the signals. Other examples include combining voice coil actuators [11] with capacitive touch screens, and small piezoceramic actuators [26, 27] attached to mobile devices. Since mobile devices already have touchscreens, these methods are designed to add tactile feedback only and do not attempt to combine the sensor and the actuator.

\section{Using vibration for sensing}

Researchers have explored using inertial sensors and pulsing vibration motors to infer interactions $[12,14]$, or to recognize tangible objects [24]. Active acoustic sensing can also be used to recognize touch on various objects and devices [25]. This approach uses a separate piezo microphone and speaker, however, to conduct a frequency sweep and thus requires adding two components to an object. Our method uses a single common component with a single frequency.

\section{Integration of touch and haptic feedback}

Recent research has also looked beyond touch screens in an attempt to integrate sensing and actuation in various interfaces. Linear motors were used in a shape display [22] to simultaneously provide haptic feedback and sense touch pressure using current measurements. Micro coils and permanent magnets were used in shiftIO $[35,34]$ to create tactile buttons that move linearly on the edge of the mobile phone. Others have explored soft pneumatically actuated materials and sensing. MorphIO, for example, integrated a silicone actuator with a conductive sponge for stretch sensing [23]. Robotics researchers have used optical [19], and inertial sensing [7] for similar purposes. 
Such integrated approaches usually involve custom and novel materials, which might be challenging to adapt on a large scale. In our approach, we use actuators that are already present in most portable electronics.

PyzoFlex is a piezo touch sensor that leverages that a charge is produced when mechanical force is applied to piezoelectrics [29]. A commercial piezo actuator and touch sensing driver chip [37] have also been developed, but the implementation details remain proprietary. Since piezoelectric actuators only produce a charge when the mechanical force is changing, it is difficult to measure pressure when the applied force is constant.

\section{Back-EMF sensing}

Back-EMF sensing is becoming increasingly widespread in brushless motors for sensorless control of the speed. It is driven by the prevalence of powerful microcontrollers, as backEMF sensing requires high processing speed. Commercial brushless motor drives, e.g., from STMicroelectronics [21], have back-EMF sensing. More recently, research demonstrated that back-EMF sensing is useful for the automatic tuning of the LRA resonance frequency $[2,5,1]$. This can compensate for changes in resonance frequency as the LRA age, which can reduce the feedback quality. Those techniques have been integrated into commercial haptic drivers, such as DRV6205 from Texas Instruments [15] and DA7280 [32] from Dialog Semi, among others. The back-EMF method has only been explored in the context of resonance tracking. To the best of our knowledge, it has not yet been explored as an interaction sensing technique.

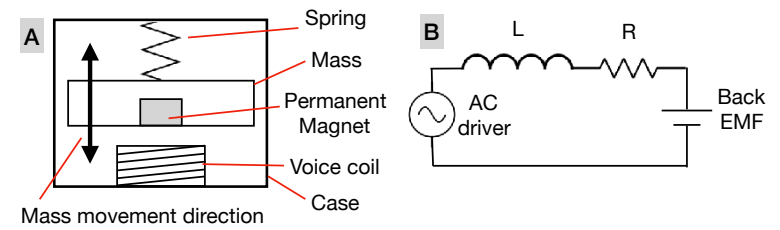

Figure 2. LRA can be simplified as a linear electric motor. A) Simplified mechanical construction of the Z-axix LRA. B) An electrical equivalent of the LRA.

\section{IMPLEMENTATION}

In this section, we describe the principles behind the operation of the LRA, such as resonance and back-EMF. We explain our design of active (LRA on) and passive sensing (LRA off), and details behind our system implementation.

\section{LRA basics}

The LRA operates as a linear electric motor and is driven by an alternating current (AC). It contains a small mass attached to a spring, as illustrated in Figure 2. To increase the haptic force, the mass-spring system is manufactured to operate at a resonance between 150 and $250 \mathrm{~Hz}$. This frequency range matches the sensitivity of the tactile receptors in our skin [16]. The mass-spring mechanical resonance frequency equation is:

$$
f=\frac{1}{2 \pi} \sqrt{\frac{k}{m}}
$$

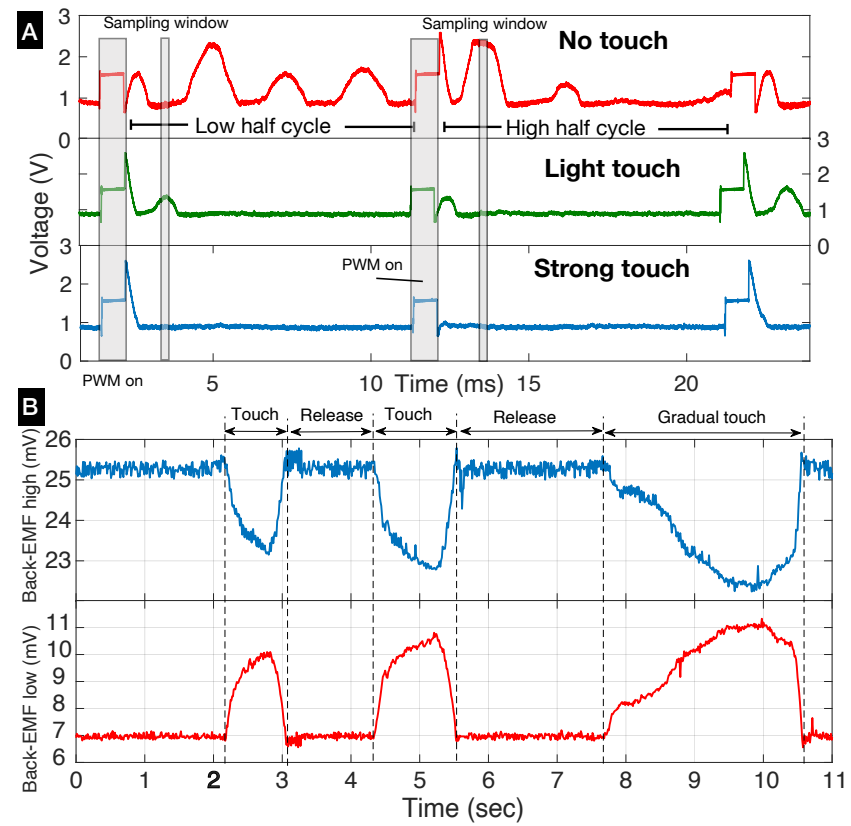

Figure 3. Sensing off-resonance. A) Close up of one cycle of amplified back-EMF voltage, after the instrumental amplifier. The oscillations of back-EMF can be seen in low and high cycles. B) Mean of sampling low and high cycles over time. This allows us to visualize the finger touch of the LRA better. Gradual touch is shown, to demonstrate the pressure sensing.

Where $k$ is the spring constant, and $m$ is the mass $(\mathrm{kg})$ attached to the spring. If an LRA is driven at an off-resonance frequency, the LRA still works, but the force is reduced.

\section{Back-EMF principles}

Back-EMF is generated in any electrical motor. The backEMF is proportional to the rate of change of magnetic flux, or in the LRA case, the velocity of the mass. Faster speed creates a higher back-EMF voltage, and stationary mass has zero back-EMF voltage. Back-EMF is governed by Lenz's law:

$$
\varepsilon=-\frac{\partial \Phi_{B}}{\partial t}
$$

Where $\varepsilon$ is the electromotive force in volts, and $\Phi_{B}$ is the magnetic flux. Magnetic flux is created as coil and magnet are moving relative to each other. Back-EMF opposes the changes in magnetic flux; thus, Lentz's law has a negative sign.

\section{Active back-EMF sensing}

Touching or contact with the LRA during vibration changes the velocity of the inside mass, as energy is dissipated into the contact object. This works well with soft viscoelastic materials such as the human body, as they absorb different amounts of energy depending on the contact force. The decrease of backEMF oscillations during touch is seen in Figure 3A.

We measure back-EMF from the floating voltage between the two LRA leads. This requires disconnecting the motor driver briefly to avoid disturbances. While the driver is disconnected, 
the mass is still oscillating inside the LRA, producing oscillating back-EMF voltage (e.g., Figure 3A). We designed a custom circuit as commercial back-EMF sensing LRA drivers do not provide the raw data.

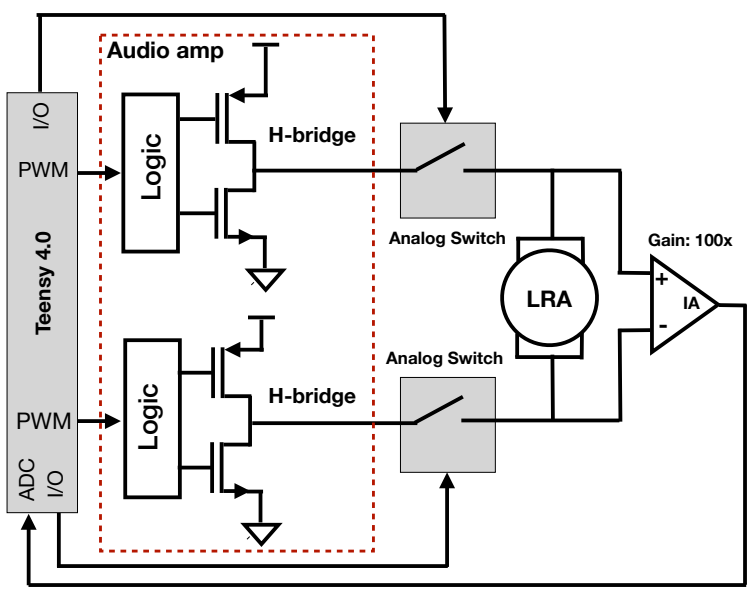

Figure 4. Simplified schematic of the LRA driver and the back-EMF measurement circuit for active sensing.

To drive the LRA, we employ an audio amplifier (MAX98306, Maxim Integrated), which contains an H-bridge output stage, as shown in Figure 4. Although this amplifier can take analog input, we use it purely for pulse input and output.

Our custom Pulse-Width-Modulation (PWM) implementation allows full waveform control and avoids the need for the digital-to-analog converters that are rare in today's microcontrollers. The H-bridge driver is employed in many commercial LRA drivers (e.g., DRV2605L) because of high energy efficiency. Also, H-bridge can reverse current, enabling electric braking for LRA. H-bridge drivers are employed in many commercial LRA drivers (e.g., DRV2605L) due to their high energy efficiency and fast braking capabilities.

We use the H-bridge driver to produce PWM output at 20 $\mathrm{kHz}$ to approximate analog waveforms. The LRA acts as a resistor-inductor (RL) low-pass filter, as shown in Figure 2. This filter converts PWM into analog waveforms for the LRA.

We use a pair of analog switches (ADG802, Analog Devices) with a maximum resistance of $0.4 \Omega$ to briefly disconnect the audio amplifier during back-EMF measurements. We can not switch it with a shutdown pin since it takes approximately 4 ms to turn it on, which would prevent the required high-speed measurements at the resonance frequency. After disconnecting from the driver, we process the back-EMF signal using an instrumental amplifier (INA114, Texas Instruments) with a gain of 500 and a $1.6 \mathrm{~V}$ reference, followed by a $50 \mathrm{ksps}$ 10-bit analog-to-digital converter (ADC). A mean of 25 data points is calculated and used for all subsequent processing. The instrumental amplifier is used to reduce the commonmode noise, such as $60 \mathrm{~Hz}$ power lines. The $60 \mathrm{~Hz}$ noise can overwhelm the signal, especially when the LRA is touched. The back-EMF voltage is small, on the order of $1-10 \mathrm{mV}$. An ARM Cortex M7 microcontroller (Teensy 4.0, PJRC) is used for digitizing and processing the signals. The microcontroller

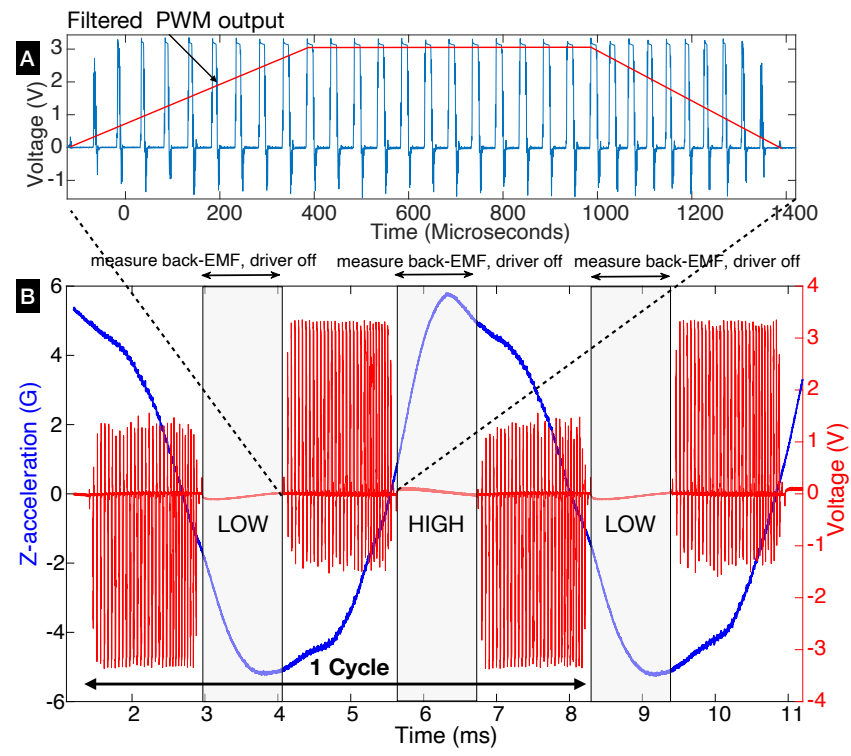

Figure 5. Example of the waveform used to drive the LRA at resonance. B) The measured acceleration (blue) is superimposed on the differential of driver's + and - outputs (red). The back-EMF was measured after negative and positive drive half-cycles, referred to as low and high. A) The top graph shows a zoomed-in view of the individual PWM drive waveform. Approximate analog filtered PWM is superimposed.

sends data to the computer over a virtual USB serial port. On the PC, the data is visualized using a Java-based application (Processing 3.0 library [10]). Also, touch and pressure sensing algorithms were implemented in the application, as well as a GUI for tuning the LRA.

\section{Active sensing waveform}

When designing sensing driving waveforms, our main considerations are audible noise, vibrations, and power consumption. Figure 5B provides an overview of the waveform's design. When using the LRA for active sensing, we do not want to cause resonance, as it creates significant vibration. When the LRA is driven at the resonant frequency, even a small applied signal is amplified to large oscillations. This is demonstrated in Figure 6, as measured by an accelerometer. As a result, we use 20 to $60 \mathrm{~Hz}$ cycle frequencies.

Figure 5A provides more detail for each individual PWM waveform. We determined the PWM shape experimentally, optimizing for capturing the smallest measurable back-EMF signal. The resulting waveforms have three equal stages: ramp up, flat, and ramp down. In our experiments, we observed that this reduced audible crackling noise, as the mass inside the LRA moved more smoothly. When the PWM is optimized for sensing, each pulse is as short as possible at $20 \mathrm{kHz}$ with a width of $1 \mathrm{us}$, which is the max resolution in our firmware's delay function. At least 10 pulses (500 us period) are required in our implementation, but this varies across LRAs, as each differs in their mechanical construction.

\section{Sensing calibration}

At resonance, the back-EMF manifests itself as a decaying oscillation. However, for sensing, the excitation signal is off- 
resonance and has low energy, resulting in aperiodic velocity and acceleration, as shown in Figure 6B. The magnitude and phase of such oscillations change when the LRA comes in contact with an object. Therefore, as shown in Figure 3A, the resulting back-EMF signal also changes magnitude and/or phase. The changes remain consistent with touch. Existing works in LRA back-EMF sensing [17, 5, 2] assume a constant phase and periodic signal at resonance, which is, however, not applicable here. Standard techniques such as the mean of the whole half-cycles do not work well, as peaks can shift, resulting in the same average. Reporting the highest peak also showed to be noisy.

In our calibration approach, first, the PWM width is adjusted to produce minimum measurable back-EMF peaks, when the LRA is touched. Then, the firmware auto-calibrates the 100 $\mu s$ ADC sampling window to the position of the highest backEMF peak. To reduce noise, this location is determined from the mode of 50 waveforms. Later, a manual adjustment of the sampling window might be needed to improve the response. The tuning has to be done only once for each setup. Our software GUI (Figure 1D) allows the display of raw values and real-time adjustment of the sampling window, as well as control of PWM (amplitude, width, frequency). This enables LRAs to be configured in real-time for sensing or actuation.

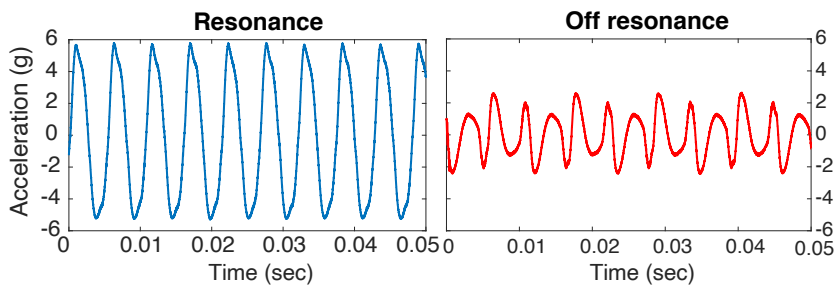

Figure 6. Comparison of acceleration of the LRA at resonance and offresonance. In both cases the LRA is driven by the same waveforms, but with frequencies of $180 \mathrm{~Hz}$ and $55 \mathrm{~Hz}$.

\section{Touch and pressure-detection algorithms}

The back-EMF readings are stored in a 25-point first-in-firstout (FIFO) buffer. To detect touch, every new reading is compared to the 10 previous readings. If the difference is higher than a configurable threshold $(65 \mathrm{mV})$, touch is registered.

Pressure sensing is enabled once touch is detected. Pressure is obtained by subtracting the current reading from the baseline. Next, pressure is linearized using the quadratic fit method described in the evaluation section. When no touch is detected, the baseline is continuously adjusted from the running average of the FIFO buffer.

\section{Passive sensing for tap detection}

The back-EMF also works oppositely as a generator. If the LRA is disturbed by an external force, voltage is produced.

Tap detection was optimized to be continuously monitored at very low power to be used as a wake-up gesture. Tap detection does not employ active sensing and thus does not need to actuate the LRA. Back-EMF voltage is generated when the LRA is tapped, as it causes movement of the magnet relative to the
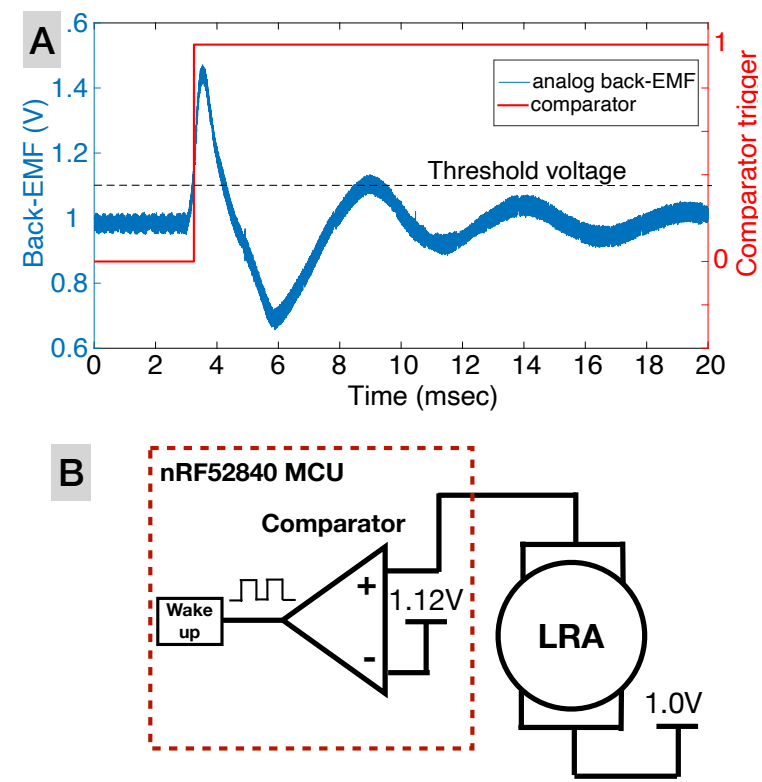

Figure 7. Tap detection using LRAs. A) Example of back-EMF produced by tapping the LRA. Also, comparator trigger output and comparator threshold voltage is shown. B) Simplified schematic diagram of the sensing circuit.

coil. An example tap capture is shown in Figure 7A. For the detection, we used a comparator with a threshold of $1.125 \mathrm{~V}$, as shown in the schematic in Figure 7B. Signal rising edge was used for triggering, and hysteresis was enabled to reduce triggering noise. The LRA was biased to $1.0 \mathrm{~V}$ to prevent negative voltage spikes that can damage the input pin. The bias could be adjusted to change sensitivity. We use a built-in low-power comparator [33] on the nRF52840 (Nordic Semiconductor) microcontroller. The microcontroller was programmed to detect interrupts produced by the comparator while in sleep mode. The passive and active LRA sensing can be combined together with 2:1 analog switches but is implemented separately here to demonstrate power optimization.

\section{Mechanical design considerations}

Mechanical mounting of the LRA is important and needs to be considered depending on the application. Furthermore, the back-EMF sensing will need to be recalibrated if mounting changes. There are two basic ways to mount the LRA. First, the simplest way is to attach the LRA directly to the chassis, e.g., using double-sided tape. In this case, the LRA becomes mechanically coupled to the object, such that they vibrate together. Mechanical coupling is usually done in mobile phones and works well if the object is rigid and has a small mass. Our back-EMF method works with coupled objects, and as we explore in the applications section, it can classify the surroundings of a mobile phone. However, it does not support touch or pressure sensing.

The second approach is to mechanically decouple the LRA from the chassis. In this case, discrete haptic feedback points from each LRA could be created, such as touch buttons with feedback or vibrotactile interfaces. Decoupling is required 
for accurate touch and pressure sensing. Such an approach requires mounting considerations to minimize coupling. The LRA should be mounted on a compliant structure. We found low-density open-cell rubber to work well.

Furthermore, to reduce noise, the LRA should not be mounted with rigid wires or connectors, as rigid objects can vibrate against each other. We found that flexible multistranded silicone wires with a diameter of $0.79 \mathrm{~mm}$ (30 AWG) produced no audible noise. Also, the wires should be in a twisted pair to reduce electrical noise during sensing.

\section{TECHNICAL EVALUATION}

In this section, we conduct technical evaluations of the LRA sensing method. We evaluate a 2.5 V LRA (G1040003D) from Jinlong Machinery and Electronics for all tests: tap, pressure, vibrations, and sound. This LRA had the highest voltage and thus produced the most force. In addition, we compare five different LRAs. To measure vibrations, we used a 3-axis analog accelerometer (ADXL326, Analog Devices), attached with double-sided tape (9628B-1-60, 3M) to the tactor. We only measured the $\mathrm{Z}$-axis acceleration.

\section{Pressure sensing}

The LRA was placed on a digital scale (SF-400C, CocinaCo) and attached with double-sided tape (9628B-1-60, 3M) to a $10 \mathrm{~mm}$ thick foam. This prevented vibration coupling to the scale. The weight measured by the scale is a proxy for applied force and touch pressure. Back-EMF and force were simultaneously recorded during finger touches. The LRA was driven off-resonance at $55 \mathrm{~Hz}$ at $3.3 \mathrm{~V}$.

The results showed that the back-EMF voltage has approximately quadratic behavior with force $\left(R^{2}=0.97\right)$. Initially, small forces create large responses in the back-EMF, and at forces around 140 grams, the back-EMF voltage saturated. This voltage-force behavior is similar to a force-sensitive resistor (FSR), which is often used for touch pressure sensing. The results indicate that this LRA is not suitable for precise force and pressure sensing. With appropriate calibration, the LRA could, however, be used to determine the approximate magnitude of a touch.

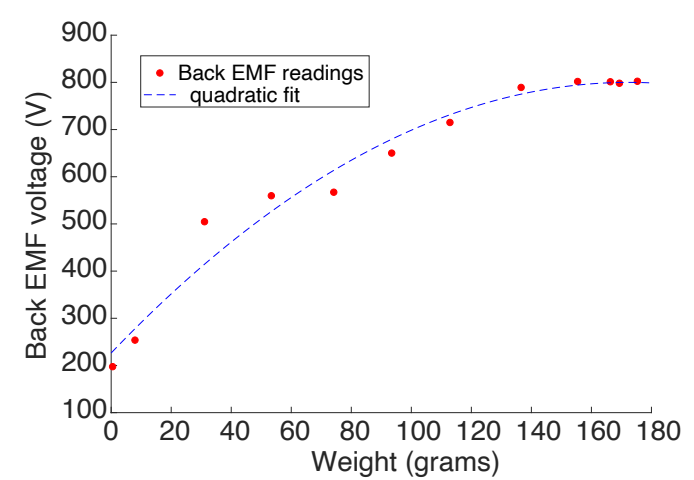

Figure 8. Back-EMF pressure sensing. Quadratic fit was applied to the data.

\section{Tap Sensitivity}

To characterize tap sensitivity, we attached an accelerometer to the LRA. We determined that an acceleration of 4.3 $\mathrm{m} / \mathrm{s}^{2}$ or higher is required for tap detection. Recall, Newton's second law states that $f=m a$, therefore using an LRA and accelerometer weight of 4.5 grams, force is $0.19 \mathrm{~N}$ or $19.4 \mathrm{gf}$ (gram-force). In comparison, tactile switches require between 50 to $1000 \mathrm{gf}$ to press [4]. The minimal required tap force corresponds to about $100 \mathrm{mV}$ back-EMF voltage. Decreasing the comparator threshold allows the detection of smaller backEMF voltage, but it increases the likelihood of false triggering due to noise. Currently, there is about $40 \mathrm{mV}$ of noise, leaving just $60 \mathrm{mV}$ headroom. If tapping is used in daily life, there is a chance of false positives if there are sudden acceleration changes (e.g., placing on hard surfaces). Methods such as double taps could be used to reduce false positives.

Part number
G0835001

Figure 9. Off-the-shelf LRAs that were evaluated for back-EMF sensing. The table describes main specifications, active and passive sensing thresholds of five LRAs.

\section{Power Consumption}

We used a digital multimeter (SDM3055, Siglent Technologies) to measure power consumption. All the reported current readings are 1 -minute mean.

For active back-EMF sensing, where the LRA needs to be driven continuously, the measured current consumption was $112.9 \mu \mathrm{A}$. However, the quiescent current consumption of the audio and differential amplifiers is significantly higher at 4.27 $\mathrm{mA}$.

Using a low-power instrumental amplifier (e.g., INA133, Texas Instruments), can reduce its power consumption from 3 $\mathrm{mA}$ to $0.2 \mathrm{~mA}$. Power gating the analog front-end can reduce power further. Running the LRA at resonance increased its current to $500 \mu \mathrm{A}$, thus showing a large improvement in power consumption with off-resonance.

Passive tap detection on an nRF52 microcontroller had a total power consumption of $37.7 \mu \mathrm{A}$ at $3.0 \mathrm{~V}$. This power consumption is promising for small, battery-powered devices. With a 225 mAh CR2032 coin cell, battery life is up to 249 days.

\section{Sound and Vibrations During Active Senisng}

The LRA's acceleration due to vibrations at resonance is 8.49 $\mathrm{m} / \mathrm{s}^{2} \mathrm{RMS}$. In comparison, active sensing acceleration is just $0.45 \mathrm{~m} / \mathrm{s}^{2} \mathrm{RMS}$. The graphical comparison is shown in Figure 6. Previous studies $[20,30]$ investigated that the threshold 
for perceptible vibration on a fingertip is between 0.05 to 0.31 $\mathrm{m} / \mathrm{s}^{2}$. The active sensing is slightly above the threshold, therefore, it could cause a minor vibrating feeling during touch.

The sound level was measured using a phone (Pixel 4, Google), using the Sound Meter app (Tools Dev). The LRA was attached to a $10 \mathrm{~mm}$ piece of foam with double-sided tape and placed 2 inches away from the phone. The mean of the background noise was recorded for 1 minute, and the LRA with pressure sensing was recorded for another minute. The LRA increased the sound level by $2 \mathrm{~dB}$. This sound level is only perceptible if the ear is in close proximity to the LRA.

\section{Comparison of five different LRAs}

Finally, we evaluated five different LRAs for compatibility with our sensing method. The results are shown in Figure 9. We were interested in the minimum PWM pulse train required to activate the different LRAs. We increased the number of PWM pulses of $1 \mu$ s on and $49 \mu$ s off until we received a measurable back-EMF during touch. We found that it varied from 300 to 2650 us. The back-EMF active touch and pressure sensing worked with all five LRAs. Although, the smaller LRAs generally required less energy (shorter min pulse). The passive tap sensing worked with Z-axis LRAs. The bias voltage had to be increased by 10 to $70 \mathrm{mV}$ for smaller-sized LRAs, as they produced a smaller back-EMF voltage. With the Y-axis LRA (ELV1411A), only tapping one side works, along the direction of motion. This LRA does not detect top taps, as with Z-axis LRAs. This creates impractical mounting, as the available tap area is very limited.
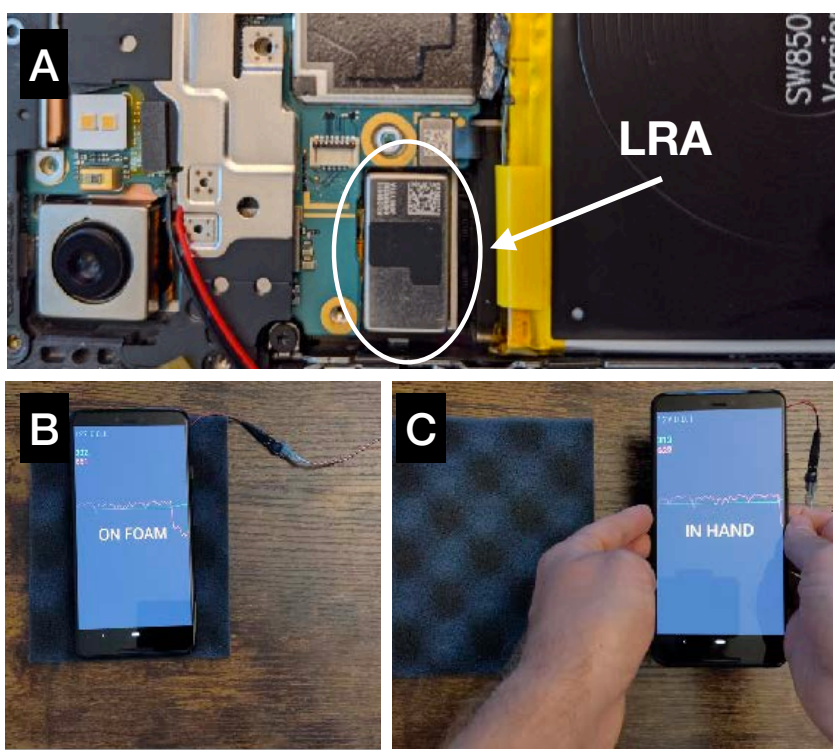

Figure 10. Using back-EMF approach with off-the-shelf devices. A) Location of the LRA, after removing the cover of the phone. B) The mobile phone's LRA used to recognize different events: placing phone on hard surface, soft surface or holding phone in hand.

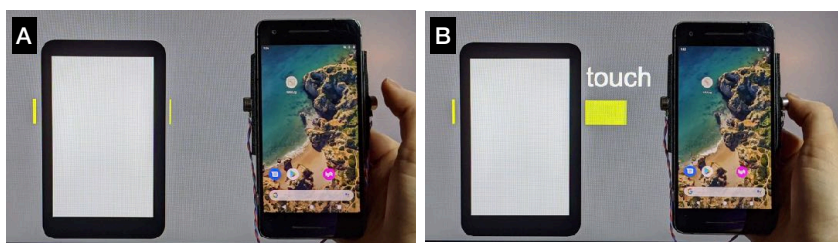

Figure 11. Two LRAs used as pressure-sensitive buttons before (A) and during touch $(\mathrm{B})$. The interface was also programmed to provide haptic feedback upon touch.

\section{APPLICATIONS}

Phone with pressure-sensing side buttons

We developed a prototype that demonstrates how LRAs could be used as combined input/output devices in portable electronics. We attached two LRAs (G1040003D), one on the left and one on the right side of a phone (Pixel 2, Google). The LRAs were mounted on a $6 \times 65 \mathrm{~mm}$ strip of $1 / 8$ in low-density rubber foam (8570K11, McMaster-Carr) with hot glue and attached to the phone using double-sided tape. The buttons provided tap, touch, and pressure sensing. They were also programmed to provide haptic feedback, once the touch was detected. This application is shown in Figure 11.

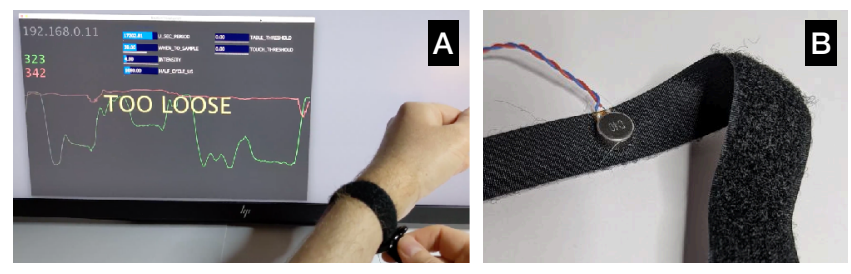

Figure 12. A tactile bracelet with the LRA facing the skin. A) The bracelet can assess whether the fit is too tight, too loose, or just right. B) Closeup of the the velco bracelet with the LRA.

\section{Haptic feedback coupling with skin}

There are a number of wearable tactile aid devices, such as sleeves [28], vests [38] and bracelets [31]. To transmit tactile feedback to the skin with consistent force, the tactor has to apply the right pressure; it can not be too loose or too tight. Currently, the only way to do so is through manual adjustment, which can be inconsistent and lacks measurable feedback. Furthermore, tactors could become loose after extended wear. We placed LRA (G1040003D) in a velcro-strap wristband. The fit was continuously monitored and prompted if its too tight, too loose, or just right, as shown in Figure 12. Potentially, this method could be used to automatically check tactor contact on a wearable tactile device and self-adjust the amplitude of the vibrotactile force.

\section{Sensing the surroundings of a mobile phone}

The LRAs used in mobile phones currently operate the same way, whether they are on a table, on a soft surface, or handheld. This causes problems, as vibrating phones slide of glass tables or emit loud and unnecessary vibrating sounds. An approach could be to stop the buzzing if the phone is picked up or not start buzzing when it already being held. Sound effects 
could also be used if the phone is vibrating on a couch or bed, to avoid ineffective vibrations. Ideally, the tactor on the phone would automatically adjust based on the sensing of its environment. To do so, the LRA can be used to detect how much vibrational energy is dissipated. We soldered wires to an LRA of a commercial mobile phone (Pixel 3XL, Google) and connected to our sensing circuit. Except for the wires, no changes were made to the phone; the LRA remained in its original place, and the phone remained functional. We use our active back-EMF technique to classify if the phone is held in hand, on a soft surface (foam), or placed on a table, as demonstrated in Figure 10. For classification, we used a hard-coded thresholding approach, but a more robust approach could utilize machine learning.

\section{LIMITATIONS AND FUTURE WORK}

The use of an LRA as the sole touch and pressure input should be carefully considered, since capacitive sensors are smaller, use less energy, are capable of proximity sensing and cost less. Furthermore, LRAs have a few disadvantages as actuators, such as limited operation frequency and size. Piezoelectric actuators could provide a viable alternative, as they are just a few $\mathrm{mm}$ thick and do not use resonance; thus, they can operate from DC to $1000 \mathrm{~Hz}$. We are therefore interested in exploring the application of our active and passive sensing for piezoelectric actuators.

Here, we only explore back-EMF-based techniques. In the future, we plan to explore other sensing techniques. For example, the current measurement could provide an alternative. Furthermore, more complex techniques, such as frequency sweep and phase differences, could characterize actuators in more detail, although they are more challenging to measure. Also, using machine learning could potentially improve the sensing and provide accurate classification of the complex back-EMF patterns.

While we focus only on sensing in this paper, our method could be developed further to enable closed-loop feedback with the actuator and sensor. This would allow the actuator to provide the same force, regardless of external conditions, such as the variable fit of a wearable device or consistent vibrations independent of the surface.

\section{CONCLUSION}

In this work, we demonstrated that off-the-shelf linear resonant actuators (LRAs) could also be used for sensing through a method that worked for all five LRA types that we evaluated. We describe three back-EMF-based sensing modalities; passive tap sensing, and active touch and pressure sensing. Passive sensing is extremely low power, running on a modern microcontroller on $37.7 \mu \mathrm{A}$. Active sensing requires powering the LRA and thus has a higher power consumption of 112.9 $\mu \mathrm{A}$, excluding sensing electronics $(4.27 \mathrm{~mA})$. We optimized the active sensing to operate off-resonance, producing only 2 $d B$ of sound and $0.45 \mathrm{~m} / \mathrm{s}^{2}$ peak-to-peak acceleration, instead of the regular $8.49 \mathrm{~m} / \mathrm{s}^{2}$.

With our applications, we show how LRA could be used as a compact, self-contained input/output device in modern wearable and mobile devices. We retrofitted a mobile phone to enable touch and pressure-sensitive side buttons. We also demonstrate that our technique can be implemented in current state-of-the-art consumer electronics, by connecting our circuit to an LRA in an off-the-shelf mobile phone. The capabilties that we enable could allow the phone to adapt to its environment by detecting if it is handheld or placed on a surface. We also demonstrate the potential for how this approach could help wearable vibrotactile devices by automatically monitoring the coupling to the skin.

We believe that this work opens new opportunities for leveraging existing ubiquitous hardware to provide rich interactions and closed-loop feedback haptic actuators.

\section{REFERENCES}

[1] Y Asai, K Hirata, and T Ota. 2012. PID feedback control method for linear resonant actuator using an estimated external load from the back-EMF as a target voltage. In International Symposium on Power Electronics Power Electronics, Electrical Drives, Automation and Motion. IEEE, 1547-1551.

[2] Yasuyoshi Asai, Katsuhiro Hirata, and Tomohiro Ota. 2013. Amplitude control method of linear resonant actuator by load estimation from the back-EMF. IEEE transactions on magnetics 49, 5 (2013), 2253-2256.

[3] Olivier Bau, Ivan Poupyrev, Ali Israr, and Chris Harrison. 2010. TeslaTouch: Electrovibration for Touch Surfaces. In Proceedings of the 23nd Annual ACM Symposium on User Interface Software and Technology (UIST '10). Association for Computing Machinery, New York, NY, USA, 283-292. DOI : http://dx.doi.org/10.1145/1866029.1866074

[4] Digikey Catalog. 2020. Tactile Switches. www.digikey. com/products/en/switches/tactile-switches/197. (2020). Accessed 2020-07-10.

[5] Youngjun Cho. 2018. Sensorless Resonance Tracking of Resonant Electromagnetic Actuator through Back-EMF Estimation for Mobile Devices. arXiv preprint arXiv:1803.07065 (2018).

[6] Inrak Choi, Heather Culbertson, Mark R. Miller, Alex Olwal, and Sean Follmer. 2017. Grabity: A Wearable Haptic Interface for Simulating Weight and Grasping in Virtual Reality. In Proceedings of the 30th Annual ACM Symposium on User Interface Software and Technology (UIST '17). Association for Computing Machinery, New York, NY, USA, 119-130. DOI : http://dx.doi.org/10.1145/3126594.3126599

[7] A. Dementyev, J. Qi, J. Ou, and J. Paradiso. 2018. Mass Manufacturing of Self-Actuating Robots: Integrating Sensors and Actuators Using Flexible Electronics. In 2018 IEEE/RSJ International Conference on Intelligent Robots and Systems (IROS). 6099-6104.

[8] Paul Dietz, William Yerazunis, and Darren Leigh. 2003. Very low-cost sensing and communication using bidirectional LEDs. In International Conference on Ubiquitous Computing. Springer, 175-191. 
[9] Taylor Dixon. 2020. Good Vibrations: How Apple Dominates the Touch Feedback Game.

https://www.ifixit.com/News/16768/

apple-taptic-engine-haptic-feedback. (2020). Accessed 2020-04-21.

[10] Processing Foundation. 2020. Processing IDE. https://processing.org/. (2020). Accessed 2020-05-01.

[11] Masaaki Fukumoto and Toshiaki Sugimura. 2001. Active Click: Tactile Feedback for Touch Panels. In CHI '01 Extended Abstracts on Human Factors in Computing Systems (CHI EA '01). Association for Computing Machinery, New York, NY, USA, 121-122. DOI : http://dx.doi.org/10.1145/634067.634141

[12] Mayank Goel, Jacob Wobbrock, and Shwetak Patel. 2012. GripSense: Using Built-in Sensors to Detect Hand Posture and Pressure on Commodity Mobile Phones. In Proceedings of the 25th Annual ACM Symposium on User Interface Software and Technology (UIST'12). Association for Computing Machinery, New York, NY, USA, 545-554. DOI :

http://dx.doi.org/10.1145/2380116.2380184

[13] Scott E. Hudson. 2004. Using Light Emitting Diode Arrays as Touch-Sensitive Input and Output Devices. In Proceedings of the 17th Annual ACM Symposium on User Interface Software and Technology (UIST '04). Association for Computing Machinery, New York, NY, USA, 287-290. DOI :

http://dx.doi.org/10.1145/1029632.1029681

[14] Sungjae Hwang, Andrea Bianchi, and Kwang-yun Wohn. 2013. VibPress: Estimating Pressure Input Using Vibration Absorption on Mobile Devices. In Proceedings of the 15th International Conference on Human-Computer Interaction with Mobile Devices and Services (MobileHCI '13). Association for Computing Machinery, New York, NY, USA, 31-34. DOI : http://dx.doi.org/10.1145/2493190.2493193

[15] Texas Instruments. 2014. DRV2605L: Haptic Driver for ERM and LRA with Built-In Library and Smart Loop Architecture. https://www.ti. com/product/DRV2605L. (2014). Accessed 2020-04-21.

[16] Lynette A Jones and Nadine B Sarter. 2008. Tactile displays: Guidance for their design and application. Human factors 50, 1 (2008), 90-111.

[17] Masayuki Kato and Katsuhiro Hirata. 2018. Characteristic Evaluation of Linear Resonant Actuator Utilizing Electrical Resonance. IEEJ Journal of Industry Applications 7, 2 (2018), 175-180.

[18] Tactile Labs. 2020. Haptuator Original. http://tactilelabs.com/products/haptics/haptuator/. (2020). Accessed 2020-04-21.

[19] Christina Larson, B Peele, S Li, S Robinson, M Totaro, L Beccai, B Mazzolai, and R Shepherd. 2016. Highly stretchable electroluminescent skin for optical signaling and tactile sensing. science 351, 6277 (2016), 1071-1074.
[20] S Maeda and MJ Griffin. 1994. A comparison of vibrotactile thresholds on the finger obtained with different equipment. Ergonomics 37, 8 (1994), 1391-1406.

[21] ST Micro. 2017. STSPIN32F0A: Advanced BLDC controller with embedded STM32 MCU. https://www.st. com/content/st_com/en/products/motor-drivers/ brushless-dc-motor-drivers/stspin32f0a.html. (2017). Accessed 2020-04-21.

[22] Ken Nakagaki, Daniel Fitzgerald, Zhiyao (John) Ma, Luke Vink, Daniel Levine, and Hiroshi Ishii. 2019. InFORCE: Bi-Directional 'Force' Shape Display for Haptic Interaction. In Proceedings of the Thirteenth International Conference on Tangible, Embedded, and Embodied Interaction (TEI '19). Association for Computing Machinery, New York, NY, USA, 615-623. DOI : http://dx.doi.org/10.1145/3294109.3295621

[23] Ryosuke Nakayama, Ryo Suzuki, Satoshi Nakamaru, Ryuma Niiyama, Yoshihiro Kawahara, and Yasuaki Kakehi. 2019. MorphIO: Entirely Soft Sensing and Actuation Modules for Programming Shape Changes through Tangible Interaction. In Proceedings of the 2019 on Designing Interactive Systems Conference (DIS '19). Association for Computing Machinery, New York, NY, USA, 975-986. DOI : http://dx.doi.org/10.1145/3322276.3322337

[24] Seungjae Oh, Gyeore Yun, Chaeyong Park, Jinsoo Kim, and Seungmoon Choi. 2019. VibEye: Vibration-Mediated Object Recognition for Tangible Interactive Applications. In Proceedings of the 2019 CHI Conference on Human Factors in Computing Systems (CHI '19). Association for Computing Machinery, New York, NY, USA, 1-12. DOI : http://dx.doi.org/10.1145/3290605.3300906

[25] Makoto Ono, Buntarou Shizuki, and Jiro Tanaka. 2013. Touch Activate: Adding Interactivity to Existing Objects Using Active Acoustic Sensing. In Proceedings of the 26th Annual ACM Symposium on User Interface Software and Technology (UIST'13). Association for Computing Machinery, New York, NY, USA, 31-40. DOI : http://dx.doi.org/10.1145/2501988.2501989

[26] Ivan Poupyrev and Shigeaki Maruyama. 2003. Tactile Interfaces for Small Touch Screens. In Proceedings of the 16th Annual ACM Symposium on User Interface Software and Technology (UIST '03). Association for Computing Machinery, New York, NY, USA, 217-220. DOI : http://dx.doi .org/10.1145/964696.964721

[27] Ivan Poupyrev, Shigeaki Maruyama, and Jun Rekimoto. 2002. Ambient Touch: Designing Tactile Interfaces for Handheld Devices. In Proceedings of the 15th Annual ACM Symposium on User Interface Software and Technology (UIST '02). Association for Computing Machinery, New York, NY, USA, 51-60. DOI : http://dx.doi.org/10.1145/571985.571993 
[28] C. M. Reed, H. Z. Tan, Z. D. Perez, E. C. Wilson, F. M. Severgnini, J. Jung, J. S. Martinez, Y. Jiao, A. Israr, F. Lau, K. Klumb, R. Turcott, and F. Abnousi. 2019. A Phonemic-Based Tactile Display for Speech Communication. IEEE Transactions on Haptics 12, 1 (2019), 2-17.

[29] Christian Rendl, Patrick Greindl, Michael Haller, Martin Zirkl, Barbara Stadlober, and Paul Hartmann. 2012. PyzoFlex: Printed Piezoelectric Pressure Sensing Foil. In Proceedings of the 25th Annual ACM Symposium on User Interface Software and Technology (UIST'12). Association for Computing Machinery, New York, NY, USA, 509-518. D0I :

http://dx.doi.org/10.1145/2380116.2380180

[30] Semin Ryu, Dongbum Pyo, Soo-Chul Lim, and Dong-Soo Kwon. 2018. Mechanical vibration influences the perception of electrovibration. Scientific reports 8,1 (2018), 1-10.

[31] Stefano Scheggi, Marco Aggravi, and Domenico Prattichizzo. 2014. A vibrotactile bracelet to improve the navigation of older adults in large and crowded environments. In Proc. 20th IMEKO TC4 Int. Symp. and 18th Int. Workshop on ADC Modelling and Testing Research on Electric and Electronic Measurement for the Economic Upturn. 798-801.

[32] Dialog Semiconductor. 2014. DA7280: Low-power high-definition haptic driver for next generation human-computer interfaces. https://www . dialog-semiconductor. com/products/haptics/da7280. (2014). Accessed 2020-04-21.
[33] Nordic Semiconductor. 2020. Infocenter: Low power comparator. https://infocenter.nordicsemi . com. (2020). Accessed 2020-05-01.

[34] Evan Strasnick and Sean Follmer. 2016. Applications of Switchable Permanent Magnetic Actuators in Shape Change and Tactile Display. In Proceedings of the 29th Annual Symposium on User Interface Software and Technology (UIST'16 Adjunct). Association for Computing Machinery, New York, NY, USA, 123-125. DOI : http://dx.doi .org/10.1145/2984751.2985728

[35] Evan Strasnick, Jackie Yang, Kesler Tanner, Alex Olwal, and Sean Follmer. 2017. ShiftIO: Reconfigurable Tactile Elements for Dynamic Affordances and Mobile Interaction. In Proceedings of the 2017 CHI Conference on Human Factors in Computing Systems (CHI '17). Association for Computing Machinery, New York, NY, USA, 5075-5086. DOI :

http://dx.doi.org/10.1145/3025453.3025988

[36] TDK. 2020. PowerHap Actuators. https://product.tdk.com/info/en/products/sw_piezo/ haptic/powerhap/index.html. (2020). Accessed 2020-04-21.

[37] Boreas Technologies. 2020. BOS1901 Piezo Haptic Driver. https:

//www.boreas.ca/products/bos1901-piezo-haptic-driver. (2020). Accessed 2020-04-21.

[38] Juan Wu, Jun Zhang, Ju Yan, Wei Liu, and Guangming Song. 2012. Design of a vibrotactile vest for contour perception. International Journal of Advanced Robotic Systems 9, 5 (2012), 166. 